\title{
The Low-redshift Intergalactic Medium
}

\author{
J. Michael Shull ${ }^{1,2}$, Steven V. Penton ${ }^{1}$ and John T. Stocke ${ }^{1}$ \\ 1 Center for Astrophysics and Space Astronomy, \\ Department of Astrophysical and Planetary Sciences, CB-389, \\ University of Colorado, Boulder, CO 80309, USA \\ 2 JILA, University of Colorado and NIST, Boulder, CO 80309, USA \\ mshull@casa.colorado.edu \\ Received 1998 November 2, accepted 1999 January 20
}

\begin{abstract}
The low-redshift Ly $\alpha$ forest of absorption lines provides a probe of large-scale baryonic structures in the intergalactic medium, some of which may be remnants of physical conditions set up during the epoch of galaxy formation. We discuss our recent Hubble Space Telescope (HST) observations and interpretation of low- $z$ Ly $\alpha$ clouds toward nearby Seyferts and QSOs, including their frequency, space density, estimated mass, association with galaxies, and contribution to $\Omega_{\mathrm{b}}$. Our HST/GHRS detections of $\sim 70$ Ly $\alpha$ absorbers with $N_{\mathrm{HI}} \geq 10^{12 \cdot 6} \mathrm{~cm}^{-2}$ along 11 sightlines covering pathlength $\Delta(c z)=114,000 \mathrm{~km} \mathrm{~s}^{-1}$ show $f\left(>N_{\mathrm{HI}}\right) \propto N_{\mathrm{HI}}{ }^{-0 \cdot 63 \pm 0 \cdot 04}$ and a line frequency $\mathrm{d} \mathcal{N} / \mathrm{d} z=200 \pm 40$ for $N_{\mathrm{HI}}>10^{12 \cdot 6} \mathrm{~cm}^{-2}$ (one every $1500 \mathrm{~km} \mathrm{~s}^{-1}$ of redshift). A group of strong absorbers toward PKS 2155-304 may be associated with gas $(400-800) h_{75}^{-1} \mathrm{kpc}$ from four large galaxies, with low metallicity $(\leq 0 \cdot 003$ solar) and $\mathrm{D} / \mathrm{H} \leq 2 \times 10^{-4}$. At low- $z$, we derive a metagalactic ionising radiation field from AGN of $J_{0}=1 \cdot 3_{-0}^{+0 \cdot 8} \times 10^{-23} \mathrm{erg} \mathrm{cm}^{-2} \mathrm{~s}^{-1} \mathrm{~Hz}^{-1} \mathrm{sr}^{-1}$ and a Ly $\alpha$-forest baryon density $\Omega_{\mathrm{b}}=(0 \cdot 008 \pm 0 \cdot 004) h_{75}^{-1}\left[J_{-23} N_{14} b_{100}\right]^{\frac{1}{2}}$ for clouds of characteristic size $b=(100 \mathrm{kpc}) b_{100}$.
\end{abstract}

Keywords: intergalactic medium-quasars: absorption lines

\section{Introduction}

Since the discovery of the high-redshift Ly $\alpha$ forest over 25 years ago, these abundant absorption features in the spectra of QSOs have been used as evolutionary probes of the intergalactic medium (IGM), galactic halos, and now large-scale structure and chemical evolution. The rapid evolution in the distribution of lines per unit redshift, $\mathrm{d} \mathcal{N} / \mathrm{d} z \propto(1+z)^{\gamma}(\gamma \approx 2 \cdot 5$ for $z \geq 1 \cdot 6)$, was consistent with a picture of these features as highly ionised 'clouds' whose numbers and sizes were controlled by the evolution of the IGM pressure, the metagalactic ionising radiation field, and galaxy formation. Early observations also suggested that Ly $\alpha$ clouds had characteristic sizes $\sim 10 \mathrm{kpc}$, were much more abundant than $\left(L_{*}\right)$ galaxies and showed little clustering in velocity space. They were interpreted as pristine, zerometallicity gas left over from the recombination era. One therefore expected low-redshift $(z<1)$ absorption clouds to show only traces of Hi, due to photoionisation and evaporation in a lower pressure IGM. All these ideas have now changed with new data and new theoretical modeling.

Absorption in the Ly $\alpha$ forest of Hi (and HeII) has long been considered an important tool for studying the high-redshift universe (Miralda-Escudé \& Ostriker 1990; Shapiro, Giroux \& Babul 1994; Fardal, Giroux \& Shull 1998). A comparison of the HI and HeII absorption lines provides constraints on the photoionising background radiation, on the history of structure formation, and on internal conditions in the Ly $\alpha$ clouds. In the past few years, these discrete Ly $\alpha$ lines have been interpreted theoretically by $N$-body hydrodynamical models (Cen et al. 1994; Hernquist et al. 1996; Zhang et al. 1997) as arising from baryon density fluctuations associated with gravitational instability during the epoch of structure formation. The effects of hydrodynamic shocks, Hubble expansion, photoelectric heating by AGN, and galactic outflows and metal enrichment from early star formation must all be considered in understanding the IGM (Shull 1998).

One of the delightful spectroscopic surprises from the Hubble Space Telescope (HST) was the discovery of Ly $\alpha$ absorption lines toward the quasar 3C 273 at $z_{\mathrm{em}}=0 \cdot 158$ by both the Faint Object Spectrograph (FOS, Bahcall et al. 1991) and the Goddard High Resolution Spectrograph (GHRS, Morris et al. 1991, 1995). In this review, we describe (Section 2) the current status of our group's long-term program with the HST and VLA to define the parameters and nature of the low-redshift $\operatorname{Ly} \alpha$ forest. In Section 3, we discuss related theoretical work on the metagalactic ionising background, $J_{\nu}(z)$, and the contribution of low- $z$ Ly $\alpha$ clouds to the baryon density, $\Omega_{\mathrm{b}}$. 


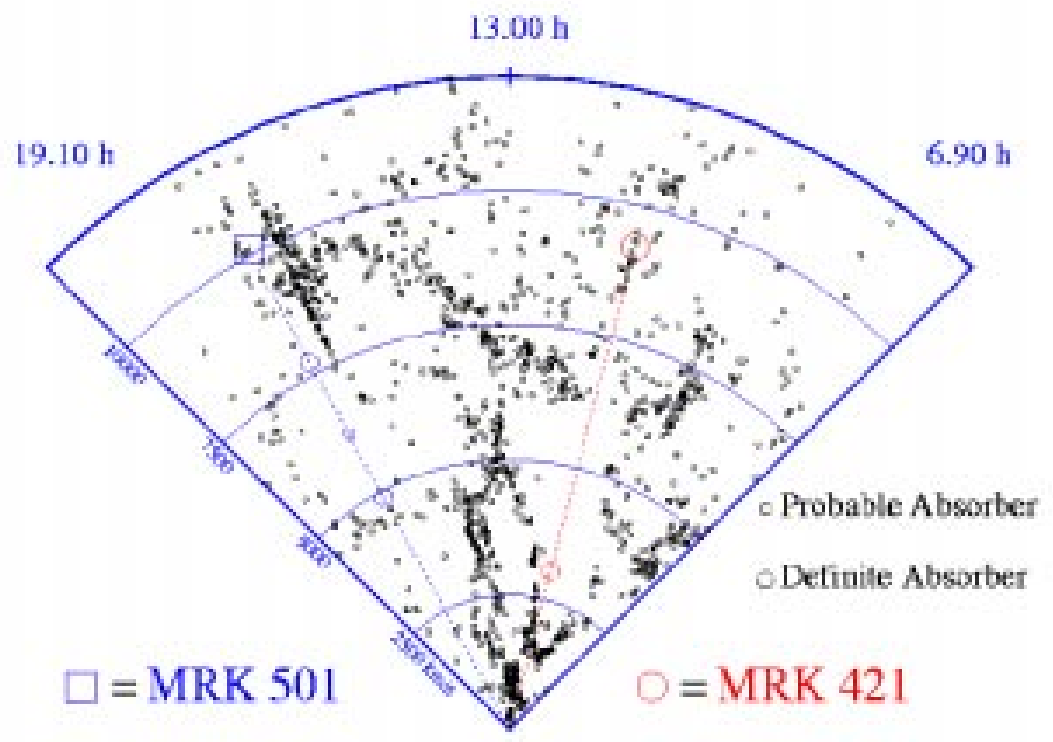

$\alpha$ wedge for $\delta-35,00 \pm 5,0^{\circ}$

Figure 1-Pie-diagram distributions of recession velocity and RA of bright (CfA survey) galaxies and four Ly $\alpha$ absorbers toward Mrk 501 and Mrk 421 (Shull, Stocke \& Penton 1996). Two of these systems lie in voids; the nearest bright galaxies lie $>4 h_{75}^{-1} \mathrm{Mpc}$ from the absorber.

\section{HST Survey of Low- $z$ Ly $\alpha$ Absorbers}

The frequency of low- $z$ Ly $\alpha$ lines with $W_{\lambda} \geq 320 \mathrm{~m} \AA$ reported by the HST/FOS Key Project, $\mathrm{d} \mathcal{N} / \mathrm{d} z=$ $(24 \cdot 3 \pm 6 \cdot 6)(1+z)^{0 \cdot 58 \pm 0 \cdot 50}$ (Bahcall et al. 1996), was considerably higher than a simple extrapolation from the high-redshift forest. These higher- $N_{\mathrm{HI}}$ absorbers exhibit associations with galaxies $\left(D \leq 200 h_{75}^{-1} \mathrm{kpc}\right)$ about half the time (Lanzetta et al. 1995).

In HST cycles 4-6, our group began GHRS studies of lower- $N_{\mathrm{HI}}$ absorbers toward 15 bright targets (Stocke et al. 1995; Shull, Stocke \& Penton 1996). These low- $z$ targets were chosen because of their well-mapped distributions of foreground galaxies (superclusters and voids). Our studies were designed to measure the distribution of $\operatorname{Ly} \alpha$ absorbers in redshift $(z \leq 0 \cdot 08)$ and column density $\left(12 \cdot 5 \leq \log N_{\mathrm{HI}} \leq 16\right)$, to probe their association with galaxies, and to measure their clustering and large-scale structure. Toward 15 targets, we detected $\sim 70$ Ly $\alpha$ systems (plus a number of highvelocity clouds and associated $\operatorname{Ly} \alpha$ absorbers) over a cumulated pathlength $c \Delta z \approx 114,000 \mathrm{~km} \mathrm{~s}^{-1}$. In cycle 7 , we will observe 14 more sightlines with the Space Telescope Imaging Spectrograph (STIS) to double our Ly $\alpha$ sample. The locations of Ly $\alpha$ absorbers toward two of our first sightlines are shown in Figure 1.

In our first four sightlines, the frequency of absorbers with $N_{\mathrm{HI}} \geq 10^{13} \mathrm{~cm}^{-2}$ was $\langle\mathrm{d} \mathcal{N} / \mathrm{d} z\rangle \approx$ $90 \pm 20$, corresponding to a local space density, $\phi_{0}=\left(0.7 \mathrm{Mpc}^{-3}\right) R_{100}^{-2} h_{75}$ for absorber radius $(100 \mathrm{kpc}) R_{100}$. This space density is $\sim 40$ times that of bright $\left(L_{*}\right)$ galaxies, but similar to that of dwarf galaxies with $L \approx 0 \cdot 01 L_{*}$. From a statistical, nearest-neighbour analysis, we found that the Ly $\alpha$ clouds have some tendency to associate with large structures of galaxies and to 'avoid the voids'. However, for the lower column systems, the nearest bright galaxies are often too far to be physically associated in hydrostatic halos or disks (Maloney 1993; Dove \& Shull 1994). Of 10 absorption systems first analysed (Shull, Stocke \& Penton 1996), three lie in voids, with the nearest bright galaxies several Mpc distant. In several cases, we identified dwarf Hi galaxies within 100-300 kpc using the VLA (Van Gorkom et al. 1996). Figure 2 shows one system toward Mrk 335, where a dwarf galaxy with $M_{\mathrm{HI}} \approx\left(7 \times 10^{7} M_{\odot}\right) h_{75}^{-2}$ and offset distance $\sim(100 \mathrm{kpc}) h_{75}^{-1}$ is seen at heliocentric velocity $c z=1955 \mathrm{~km} \mathrm{~s}^{-1}$, remarkably near to that of the Ly $\alpha$ absorber. Thus, some of the lower- $N_{\mathrm{HI}}$ absorbers appear to be associated with dwarf galaxies, although much better statistics are needed.

In HST cycle 6 , we observed seven more sightlines with the GHRS/G160M. With better data, we were able to detect weaker Ly $\alpha$ absorption lines, down to $20 \mathrm{~m} \AA\left(N_{\mathrm{HI}}=10^{12 \cdot 6} \mathrm{~cm}^{-2}\right)$ in some cases. Many of the new sightlines exhibit considerably more Ly $\alpha$ absorbers; for these 15 sightlines, $\langle\mathrm{d} \mathcal{N} / \mathrm{d} z\rangle=200 \pm 40$ for $N_{\mathrm{HI}} \geq 10^{12 \cdot 6} \mathrm{~cm}^{-2}$ or one line every $1500 \mathrm{~km} \mathrm{~s}^{-1}$. Although there is wide variation, this frequency is over twice the value (one every $3400 \mathrm{~km} \mathrm{~s}^{-1}$ ) reported earlier (Shull, Stocke \& Penton 1996) for $N_{\mathrm{HI}} \geq 10^{13}$ 
$\mathrm{cm}^{-2}$. For a curve of growth with $b=25 \mathrm{~km} \mathrm{~s}^{-1}$, the 70 Ly $\alpha$ absorbers with $12 \cdot 6 \leq \log N_{\mathrm{HI}} \leq 14 \cdot 0$ follow a distribution $f\left(\geq N_{\mathrm{HI}}\right) \propto N_{\mathrm{HI}}^{-0} \cdot 63 \pm 0 \cdot 04$, remarkably close to the slope in the high- $z$ Ly $\alpha$ forest. These results have been corrected for incompleteness at low equivalent widths, for line blending, and for the GHRS sensitivity function versus wavelength (Penton, Stocke \& Shull 1999).

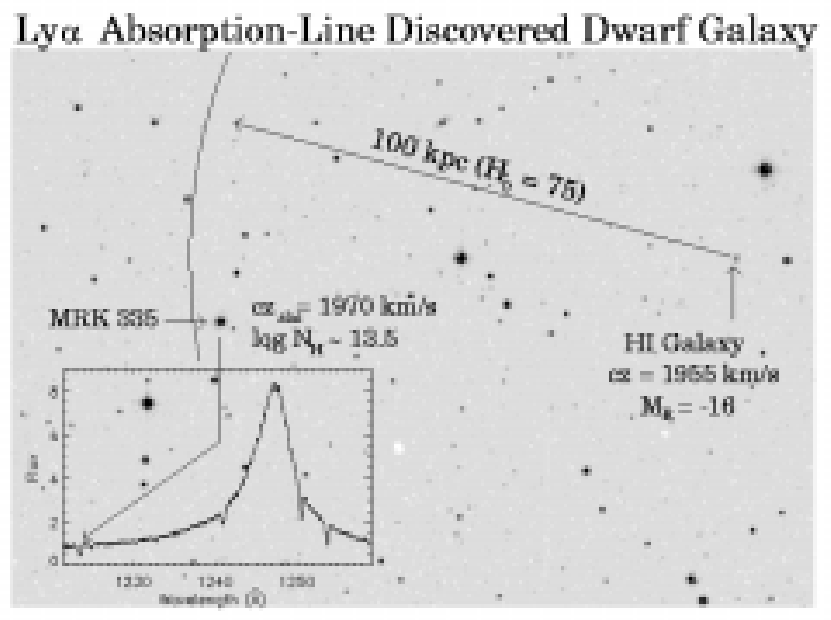

Figure 2-Overlay of galaxy field around Mrk 335, showing a dwarf galaxy at $1955 \mathrm{~km} \mathrm{~s}^{-1}$ at nearly the same redshift as the $1970 \mathrm{~km} \mathrm{~s}^{-1}$ Ly $\alpha$ absorber $\left(N_{\mathrm{HI}}=10^{13 \cdot 5} \mathrm{~cm}^{-2}\right)$. The offset distance is $\sim 95 h_{75}^{-1} \mathrm{kpc}$.
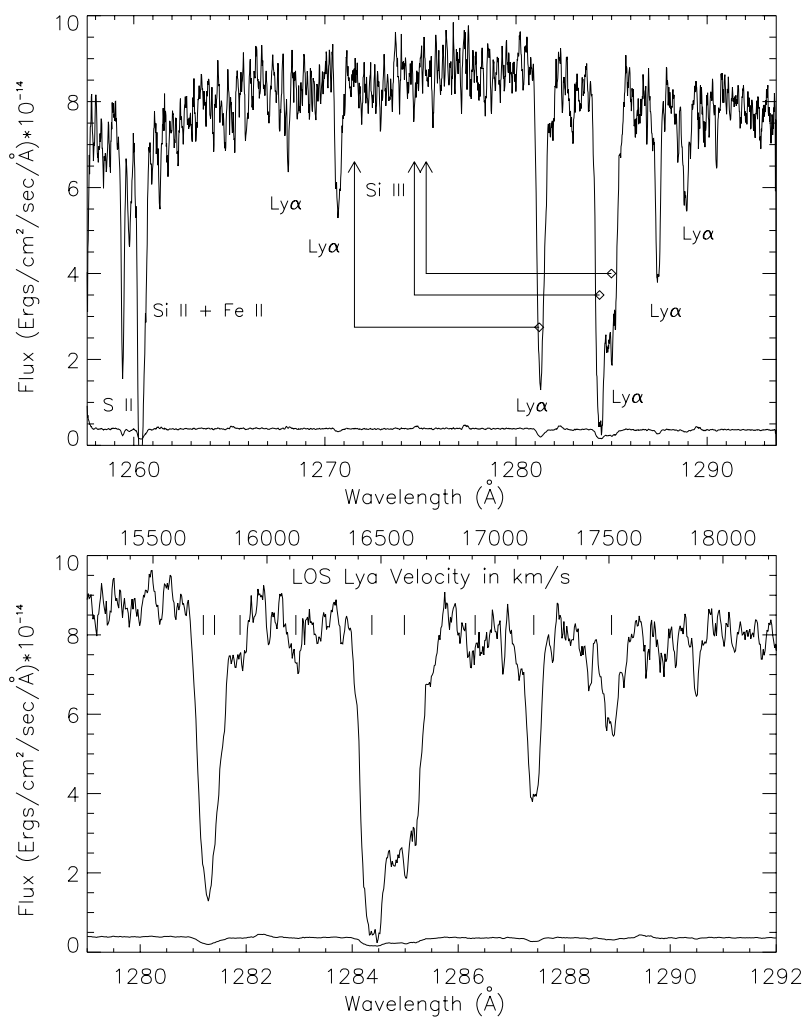

Figure 3-HST/GHRS (G160M) spectrum of PKS 2155-304 (Shull et al. 1998) shows multiple Ly $\alpha$ absorption systems between 1281-1290 $\AA\left(c z=15,700-17,500 \mathrm{~km} \mathrm{~s}^{-1}\right)$. Upper limits on Si III $\lambda 1206 \cdot 50$ absorption at $1274 \cdot 7 \AA$ and $1275 \cdot 2 \AA$ correspond to $[\mathrm{Si} / \mathrm{H}] \leq 0 \cdot 003$ solar abundance.
We turn now to the extraordinary sightline toward PKS 2155-304 (Bruhweiler et al. 1993; Shull et al. 1998). This target exhibits numerous Ly $\alpha$ absorbers (Figure 3), including a group of strong systems between $c z=15,700$ and $17,500 \mathrm{~km} \mathrm{~s}^{-1}$. The strong absorbers have an estimated combined column density $N_{\mathrm{HI}}=(2-5) \times 10^{16} \mathrm{~cm}^{-2}$, based on Lymanlimit absorption seen by ORFEUS (Appenzeller et al. 1995). Using the VLA (Van Gorkom et al. 1996; Shull et al. 1998), we have identified these absorbers with the very extended halos or intragroup gas associated with four large galaxies at the same redshift (Figure 4). The offsets from the sightline to these galaxies are enormous. Despite the kinematic associations, it would be challenging to make a dynamical association with such galaxies. One must extrapolate from the $10^{20} \mathrm{~cm}^{-2}$ columns seen in galactic $21-\mathrm{cm}$ emission to the range $10^{13-16} \mathrm{~cm}^{-2}$ probed by Ly $\alpha$ absorption. Much of the strong Ly $\alpha$ absorption may arise in gas of wide extent, $\sim 1 \mathrm{Mpc}$ in diameter, spread throughout the group of galaxies at $z=0 \cdot 057$. Assuming that $\left\langle N_{\mathrm{HI}}\right\rangle \approx 2 \times 10^{16} \mathrm{~cm}^{-2}$ and applying corrections for ionisation $\left(\mathrm{H}^{\circ} / \mathrm{H} \approx 3 \times 10^{-4}\right.$ for $J_{0}=10^{-23}$ and $600 \mathrm{kpc}$ cloud depth) and for helium mass $(Y=0 \cdot 24)$, the gas mass could total $\sim 10^{12} M_{\odot}$.

These absorbers offer an excellent opportunity to set stringent limits on heavy-element abundances and $\mathrm{D} / \mathrm{H}$ in low-metallicity gas in the far regions of such galaxies. For example, no Si III $\lambda 1206 \cdot 50$ absorption is detected (rest equivalent width $W_{\lambda} \leq 22$ $\mathrm{m} \AA$ or $N_{\mathrm{SiIII}} \leq 1 \cdot 0 \times 10^{12} \mathrm{~cm}^{-2}$ at $\left.4 \sigma\right)$ at wavelengths corresponding to the strong Ly $\alpha$ absorbers near 1281 $\AA$ and $1285 \AA$. Over a range of photoionisation models for $\left(\mathrm{H}^{\circ} / \mathrm{H}\right)$ and $\left(\mathrm{Si}^{+2} / \mathrm{Si}\right)$, this limit corresponds to an abundance $(\mathrm{Si} / \mathrm{H}) \leq 0 \cdot 003(\mathrm{Si} / \mathrm{H})_{\odot}$ for an assumed $N_{\mathrm{HI}}=2 \times 10^{16} \mathrm{~cm}^{-2}$ and $300-600 \mathrm{kpc}$ cloud depth (Shull et al. 1998). The lack of observed C IV $\lambda 1549$ absorption leads to similar limits, [C/H] $<0.005$ solar. A rudimentary analysis of the lack of observed D I ( $\mathrm{Ly} \alpha)$ absorption in the blueward wings of the strong $\mathrm{HI}$ lines suggests that $(\mathrm{D} / \mathrm{H})$ $\leq 2 \times 10^{-4}$. These limits can be improved with more sophisticated profile fitting and future data from HST/STIS (cycle 8) and FUSE.

The Hi toward PKS 2155-304 appears to represent gas with the lowest detected metallicity. Was this gas was once inside the galaxies at $c z=17,000 \pm 1000$ $\mathrm{km} \mathrm{s}^{-1}$, or is it pristine? We can perhaps answer this question by deeper spectral searches for traces of metals. The origin of the lower-column Ly $\alpha$ systems would seem to be more diverse, possibly arising in extended halos or debris disks of dwarf galaxies, large galaxies, and small groups (Morris \& van den Bergh 1994).

\section{Theoretical Implications}

A primary theoretical issue is whether low- $z$ clouds have any relation to the evolution of the baryons in 


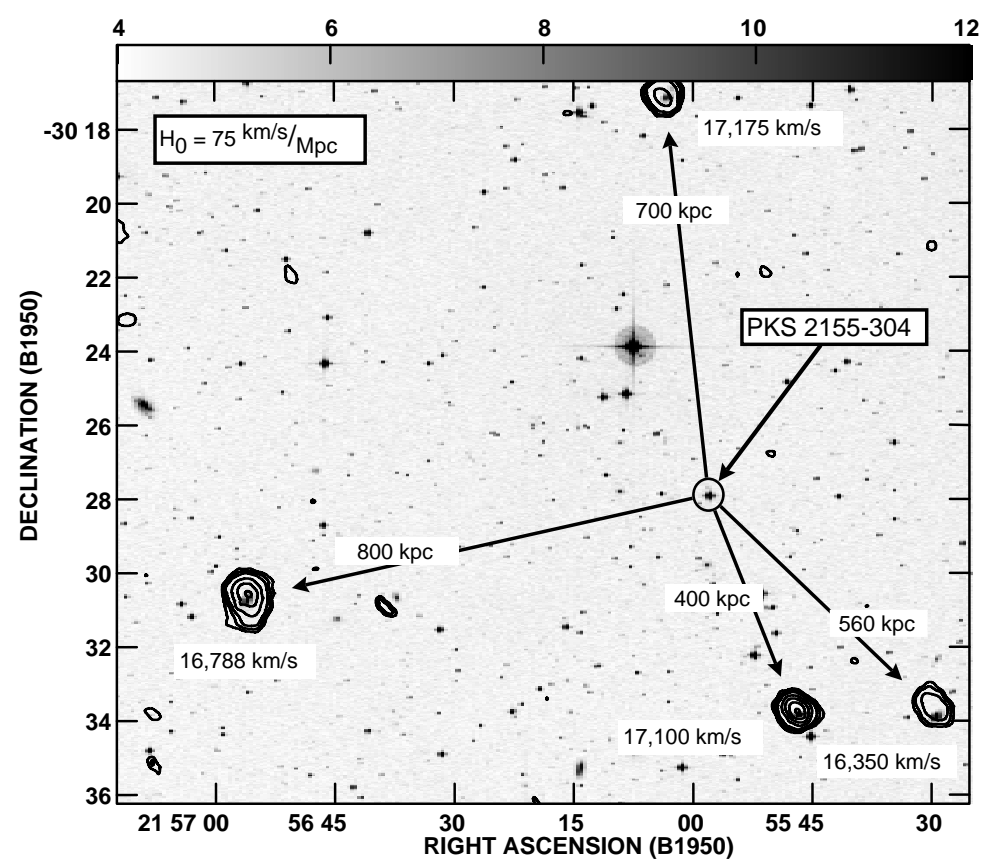

Figure 4-VLA field of 21-cm emission toward PKS 2155-304 at velocities $16,000-17,300 \mathrm{~km} \mathrm{~s}^{-1}$ near the Ly $\alpha$ absorbers. Four large Hi galaxies are detected at projected offsets of $(400-800) h_{75}^{-1} \mathrm{kpc}$ (Shull et al. 1998). At least two galaxies, to the south and southwest, appear to be kinematically associated with Ly $\alpha$ absorbers at 16,460 and $17,170 \mathrm{~km} \mathrm{~s}^{-1}$.

the high- $z$ forest. A quick estimate suggests that the low- $z$ absorbers could contain a substantial (25\%) fraction of the total baryons estimated from Big Bang nucleosynthesis, $\Omega_{\mathrm{BBN}}=(0 \cdot 0343 \pm 0 \cdot 0025) h_{75}^{-2}$ (Burles \& Tytler 1998). Consider those Ly $\alpha$ systems with $N_{\mathrm{HI}} \geq 10^{13} \mathrm{~cm}^{-2}$, for which one can derive the space density $\phi_{0}$,

$$
\frac{\mathrm{d} \mathcal{N}}{\mathrm{d} z}=\phi_{0}\left(\pi R_{0}^{2}\right) \frac{c}{H_{0}} \approx 100 .
$$

The major uncertainty in deriving absorber masses is the ionisation correction, which depends on the profile of gas density around the cloud centres. Assume, for simplicity, that $n_{\mathrm{H}}(r)=$ $n_{0}\left(r / r_{0}\right)^{-2}$ and adopt photoionisation equilibrium with photoionisation rate $\Gamma_{\mathrm{HI}}$ and a case- $\mathrm{A}$ hydrogen recombination rate coefficient, $\alpha_{\mathrm{H}}^{(\mathrm{A})}$, at 20,000 K. The ionising radiation field is $J_{\nu}=J_{0}\left(\nu / \nu_{0}\right)^{-\alpha_{s}}$ with $\alpha_{s} \approx$ $1 \cdot 8$ and $J_{0}=\left(10^{-23} \mathrm{erg} \mathrm{cm}^{-2} \mathrm{~s}^{-1} \mathrm{~Hz}^{-1} \mathrm{sr}^{-1}\right) J_{-23}$. The Hi column density integrated through the cloud at impact parameter $b$ is

$$
N_{\mathrm{HI}}(b)=\frac{\pi n_{0}^{2} r_{0}^{4} \alpha_{H}^{(A)}\left(1+2 n_{\mathrm{He}} / n_{\mathrm{H}}\right)}{2 \Gamma_{\mathrm{HI}} b^{3}} .
$$

We can solve for $n_{0} r_{0}^{2}$ and find the total gas mass within $b=(100 \mathrm{kpc}) b_{100}$ for a fiducial column density $N_{\mathrm{HI}}=\left(10^{14} \mathrm{~cm}^{-2}\right) N_{14}$,

$$
\begin{aligned}
M_{\mathrm{cl}}(b) & =\left[4 \pi n_{0} r_{0}^{2} b\left(1 \cdot 22 m_{\mathrm{H}}\right)\right] \\
& =\left(1 \cdot 6 \times 10^{9} M_{\odot}\right) N_{14}^{\frac{1}{2}} J_{-23}^{\frac{1}{2}} b_{100}^{\frac{5}{2}},
\end{aligned}
$$

which yields a cloud closure parameter in baryons,

$$
\begin{aligned}
\Omega_{\mathrm{b}} & \approx \phi_{0}(b) M_{\mathrm{cl}}(b) \\
& =(0 \cdot 008 \pm 0 \cdot 004) J_{-23}^{\frac{1}{2}} N_{14}^{\frac{1}{2}} b_{100}^{\frac{1}{2}} h_{75}^{-1} .
\end{aligned}
$$

For the spherical-cloud model, the radiation field, cloud size, and column-density distribution probably each contribute $30-40 \%$ to the uncertainty in $\Omega_{\mathrm{b}}$, while temperature $T_{\mathrm{e}}$ and ionising spectral index $\alpha_{\mathrm{s}}$ contribute $10 \%$, for an overall uncertainty of $50 \%$. However, as with the high- $z$ forest, the greatest uncertainty in $\Omega_{\mathrm{b}}$ lies in the cloud geometry and radial profile. These parameters can only be understood by building up statistics through many sightlines, particularly multiple targets that probe the same cloud structures.

We have also increased our understanding of the metagalactic ionising background radiation and the 'Gunn-Peterson' opacities, $\tau_{\mathrm{HI}}(z)$ and $\tau_{\mathrm{HeII}}(z)$. Using a new cosmological radiative transfer code and IGM opacity model, Fardal, Giroux \& Shull (1998) modelled the ionisation fractions of $\mathrm{HI}_{\mathrm{I}}$ and HeII in a fluctuating radiation field due to quasars and starburst galaxies. In this work, we have calculated the metagalactic ionising radiation field, $J_{\nu}(z)$, using QSO and stellar emissivities and including cloud diffuse emission and new (somewhat lower) IGM opacities derived from Keck Ly $\alpha$ forest spectra.

Figure 5 illustrates the evolution of $J_{\nu}$ from $z=5 \rightarrow 0$, peaking at $z \approx 3$. At $z<2$, the absorption breaks at 1 Ryd (HI) and 4 Ryd (HeII) become much less prominent and $J_{\nu}$ drops rapidly. 


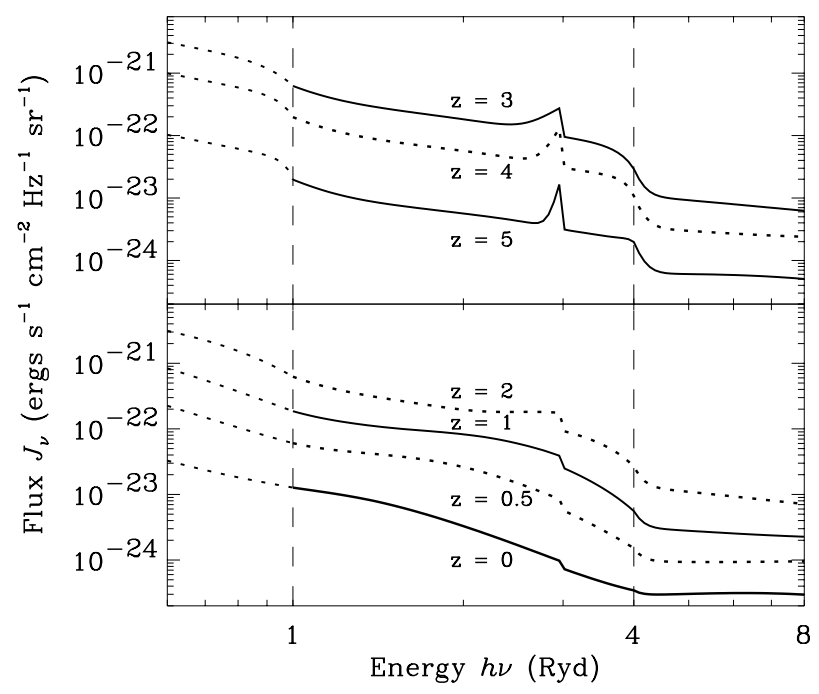

Figure 5-Spectrum $J_{\nu}(z)$ of ionising background from redshift $z=5 \rightarrow 0$ from new opacity and radiative transfer models (Fardal, Giroux, \& Shull 1998; Shull et al. 1999).

At low redshift $(z<0 \cdot 5), J_{\nu}$ depends both on the local (Seyfert) luminosity function and on the opacity model. We have recomputed the ionising radiation field at $z \approx 0$ (Shull et al. 1999) using a new opacity model from HST absorption data and extrapolated EUV emissivities of QSOs and low-redshift Seyferts from our IUE-AGN database (Penton \& Shull, unpublished). We find $J_{0}=\left(1 \cdot 3_{-0}^{+0 \cdot 5}\right) \times 10^{-23} \mathrm{erg}$ $\mathrm{cm}^{-2} \mathrm{~s}^{-1} \mathrm{~Hz}^{-1} \mathrm{sr}^{-1}$ at $z=0$, very close to our adopted scaling parameter, $J_{-23}=1$. We clearly still have an enormous amount to learn about the nature and distribution of the low-redshift Ly $\alpha$ clouds. It seems likely that future studies may uncover valuable information about their connection to large-scale structure and to the processes of galaxy formation and evolution.

\section{Acknowledgments}

Our Ly $\alpha$ observations were made with the NASA/ESA Hubble Space Telescope supported by grant GO06586 01-95A through the Space Telescope Science
Institute. Theoretical work was supported by NSF grant AST96-17073. Our colleagues in the VLA studies are Jacqueline Van Gorkom (Columbia University) and Chris Carilli (NRAO). Theoretical work was done in collaboration with Mark Giroux and Mark Fardal (Colorado) and undergraduate research student David Roberts (Cornell).

\section{References}

Appenzeller, I., Mandel, H., Krautter, J., Bowyer, S., Hurwitz, M., Grewing, M., Kramer, G., \& Kappelmann, N. 1995, ApJ, 439, L33

Bahcall, J. N., Januzzi, B. T., Schneider, D. P., Hartig, G. F., Bohlin, R., \& Junkkarinen, V. 1991, ApJ, 377, L5

Bahcall, J. N., et al. 1996, ApJ, 457, 19

Bruhweiler, F. C., Boggess, A., Norman, D. J., Grady, C. A., Urry, C. M., \& Kondo, Y. 1993, ApJ, 409, 199

Burles, S., \& Tytler, D. 1998, ApJ, 499, 699

Cen, R., Miralda-Escudé, J., Ostriker, J. P., \& Rauch, M. 1994, ApJ, 437, L9

Dove, J. B., \& Shull, J. M. 1994, ApJ, 423, 196

Fardal, M. A., Giroux, M. L., \& Shull, J. M. 1998, AJ, 115, 2206

Hernquist, L., Katz, N., Weinberg, D. H., \& Miralda-Escudé, J. 1996, ApJ, 457, L51

Lanzetta, K. M., Bowen, D. V., Tytler, D., \& Webb, J. K. 1995, ApJ, 442, 538

Maloney, P. 1993, ApJ, 414, 41

Miralda-Escudé, J., \& Ostriker, J. P. 1990, ApJ, 350, 1

Morris, S. L., \& van den Bergh, S. 1994, ApJ, 427, 696

Morris, S. L., Weymann, R. J., Savage, B. D., \& Gilliland, R. L. 1991, ApJ, 377, L21

Morris, S. L., et al. 1995, ApJ, 419, 524

Penton, S., Stocke, J. T., \& Shull, J. M. 1999, in preparation

Shapiro, P., Giroux, M., \& Babul, A. 1994, ApJ, 427, 25

Shull, J. M. 1998, Nature, 394, 17

Shull, J. M., Penton, S., Stocke, J. T., Giroux, M. L., Van Gorkom, J. H., \& Carilli, C. 1998, AJ, 116, 2094

Shull, J. M., Roberts, D., Giroux, M. L., Penton, S., \& Fardal, M. A. 1999, AJ, submitted

Shull, J. M., Stocke, J. T., \& Penton, S. 1996, AJ, 111, 72

Stocke, J. T., Shull, J. M., Penton, S., Donahue, M., \& Carilli, C. 1995, ApJ, 451, 24

Van Gorkom, J. H., Carilli, C. L., Stocke, J. T., Perlman, E. S., \& Shull, J. M. 1996, AJ, 112, 1397

Zhang, Y., Meiksin, A., Anninos, P., \& Norman, M. 1997, ApJ, 495, 63 